\title{
Guest editorial: spatial and temporal databases
}

\author{
Dieter Pfoser • Yufei Tao • Guest Editors
}

Published online: 23 May 2013

(C) Springer Science+Business Media New York 2013

With the emergence of spatial and temporal data being important structuring elements of today's Internet, this special issue presents some outstanding research results that address novel indexing and query processing challenges. This issue consists of three papers, which are briefly discussed as follows.

The first paper of this special issue is entitled "FAST: A Generic Framework for Flash-Aware Spatial Trees" and is written by Mohamed Sarwat, Mohamed Mokbel, Xun Zhou (all University of Minnesota), and Suman Nath (Microsoft Research). The paper presents a general technique for converting a traditional disk-oriented structure to an access method that works well on flash-memory devices. Applicable to several well-known structures (including the B- and R-trees), the technique aims at achieving two purposes simultaneously: (i) minimizing the update and query overhead, and (ii) preventing the loss of data even in a system crash, thus ensuring data durability. The paper contains several novel ideas, which are of independent interests since they may also be useful in designing other flash-aware algorithms. In addition, the paper features a real system that implements the proposed technique and is demonstrated to have excellent performance in practice through extensive experiments.

The second paper, "Inverse Queries for Multidimensional Spaces" by Andreas Zuefle, Tobias Emrich, Hans-Peter Kriegel, Matthias Renz (all Ludwig-Maximilians-Universität München), Nikos Mamoulis, Shiming Zhang (University of Hong Kong) and Thomas Bernecker (Ludwig-Maximilians-Universität München) proposes a new class of problems called inverse search. Given a set $\mathrm{S}$ of objects, the goal of inverse search is to find which queries (among a large pool of candidate queries) have $\mathrm{S}$ in their results. It can be integrated with various query definitions, e.g., range searching, $\mathrm{k}$ nearest neighbor search, dynamic skyline searching, etc.; and in each case, inverse search specializes into a specific, well-

Dieter Pfoser is on leave from "ATHENA" Research Center, Marousi, Greece.

D. Pfoser $(\bowtie)$

Department of Geography and Geoinformation Science, George Mason University, Fairfax, VA, USA

e-mail: dpfoser@gmu.edu

Y. Tao

Department of Computer Science and Engineering, Chinese University of Hong Kong, Hong Kong, China e-mail: taoyf@cse.cuhk.edu.hk 
defined, retrieval problem that is often rather challenging to tackle. The paper presents a general framework for performing inverse search, and then, gives concrete algorithms under the framework to solve the inverse search problem for all the query definitions mentioned earlier. Extensive experiments confirm the efficiency of the proposed algorithms. Besides proposing a novel problem and providing solutions with very good technical depth, the paper also has the potential of attracting further research (on inverse search coupled with other query definitions).

The third paper is entitled "MIDAS: Multi-Attribute Indexing for Distributed Architecture Systems" and is written by George Tsatsanifos (National Technical University of Athens), Dimitris Sacharidis and Timos Sellis (both "ATHENA" Research Center, Greece). This paper presents a novel multi-dimensional indexing infrastructure for highly dynamic P2P networks. The approach adapts a randomized adaptive kd-tree as the underlying index within a P2P overlay. Peers are responsible for the leaves and keep $\mathrm{O}(\log \mathrm{n})$ additional information about its path, its neighbors along the path and its inverse neighbors (backlinks). Overall the approach presents a cleanly designed P2P index that inherits many nice properties from randomized binary trees.

We hope that you will enjoy reading this special selection and we are grateful to GeoInformatica for giving us the opportunity to publish these high quality articles. Here, we would also like to thank the authors and referees for their effort in organizing this special issue.

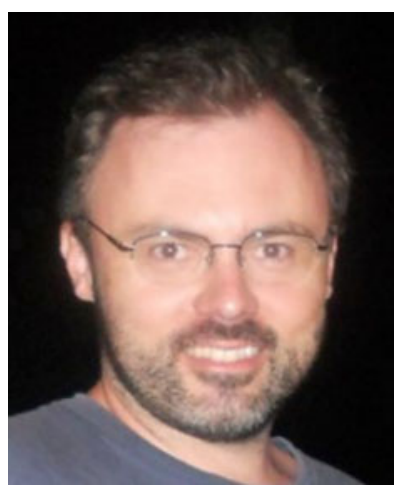

Dieter Pfoser is an associate professor at the Department of Geography and Geoinformation Science, George Mason University, USA and a senior researcher at the "ATHENA" Research Center, Greece (on leave). He is an editorial board member of Transactions in GIS. He is/was a PC co-chair of W2GIS 2014, organizer of the 2nd SIGSPATIAL GEOCROWD Workshop 2013, a PC co-chair of SSTD 2011, and a senior PC member of the ACM SIGSPATIAL GIS conference 2012, 2013. 


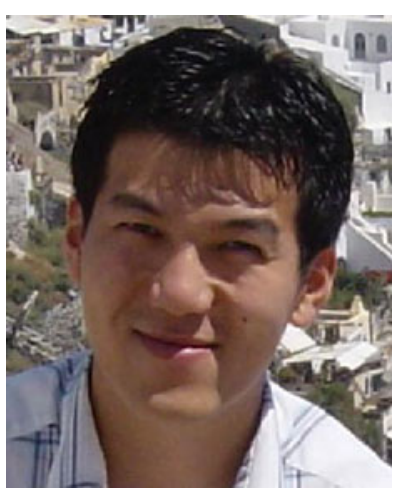

Yufei Tao is a full professor at the Chinese University of Hong Kong. He also holds a Visiting Professor position, under the World Class University (WCU) program of the Korean government, at the Korea Advanced Institute of Science and Technology (KAIST). He is an associate editor of ACM Transactions on Database Systems (TODS), and of IEEE Transactions on Knowledge and Data Engineering (TKDE). He is/ was a PC co-chair of ICDE 2014, a PC co-chair of SSTD 2011, an area PC chair of ICDE 2011, and a senior PC member of CIKM 2010, 2011, 2012. 